\title{
Prevención del suicidio y la conducta suícida
}

\author{
Carlos Campillo Serrano* y Germán Fajardo Dolci
}

Facultad de Medicina, Universidad Nacional de México, Ciudad de México, México

\section{Resumen}

En México la conducta suicida es una preocupación porque, aunque las tasas de suicidio consumado están por debajo de la media mundial (11.4 vs. 4.1 x 100,000 habitantes), entre 1970 y 2007 aumentaron un 175\%, sobre todo a expensas de los jóvenes, en los cuales es la tercera causa de muerte. Por tal motivo, se han elaborado varias acciones preventivas que no han tenido el éxito esperado, porque están mal planteadas. Carecen de una definiciones de caso uniforme y clara. Su enfoque es reduccionista, se apega solo al de la salud pública y no salen del esquema tradicional de prevención primaria, secundaria y terciaria. La presente revisión pretende ofrecer una opciones diferentes. Adoptar como definición de caso la de los Centros para el Control y la Prevención de la Enfermedades (CCD) de Atlanta. Ampliar la visión incluyendo aspectos filosóficos, psicosociales y psiquiátricos. Incluir como marco teórico el modelo psicológico «diátesis-estrés» y proponer como estrategia preventiva «las intervenciones por poblaciones».

PALABRAS CLAVE: Suicidio. Conducta suicida. Prevención.

\section{Suicide prevention and suicidal behavior}

\section{Abstract}

In Mexico, suicidal behavior is a matter of concern because, although the rates of death by suicide are below global mean (11.4 vs. 4.1 x 100,000 population), between 1970 and 2007 they increased by $175 \%$, especially among young people, in whom it is the third leading cause of death. For this reason, several preventive actions have been developed but have not had the expected success, because they are poorly designed. They lack a uniform and clear case definition. Their approach is reductionist, it focuses only on the public health aspect and does not go beyond traditional plans of primary, secondary and tertiary prevention. This review aims to offer different options, such as adopting the case definition provided by Atlanta's Centers for Disease Control and Prevention (CDC). It aims to broaden the vision in order to include philosophical, psychosocial and psychiatric aspects, as well as to include, as a theoretical framework, the "diathesis-stress" psychological model and propose a population-based intervention preventive strategy.

KEY WORDS: Suicide. Suicidal behavior. Prevention.

\section{Introducción}

El suicidio y la conducta suicida son fenómenos universales. Se observa en todas las regiones y las culturas. La Organización Mundial de la Salud estimó que en 2012 en el mundo se suicidaron 804,000 personas. Cada 40 segundos se suicida una persona en el mundo'. En México las tasas de suicidio consumado están por debajo de la media mundial (11.4 vs. 4.1 por cada cien mil habitantes) ${ }^{2}$. Aun así se ha convertido en un problema importante de salud publica, porque entre 1970 y 2007 las tasas subieron un 175\%,
Correspondencia:

${ }^{*}$ Carlos Campillo-Serrano

E-mail: ccs1944@gmail.com
Fecha de recepción: 08-04-2021

Fecha de aceptación: 08-07-2021

DOI: 10.24875/GMM.21000205
Gac Med Mex. 2021;157:564-569

Disponible en PubMed

www.gacetamedicademexico.com

0016-3813/@ 2021 Academia Nacional de Medicina de México, A.C. Publicado por Permanyer. Este es un artículo open access bajo la licencia CC BY-NC-ND (http://creativecommons.org/licenses/by-nc-nd/4.0/). 
a expensas de los jóvenes, entre quienes es ya la tercera causa de muerte ${ }^{3,4}$. Si bien se han propuesto diferentes programas preventivos, estos, lamentablemente, no han prosperado, pese a que el suicidio es uno de los pocos temas de la salud mental que puede contar con intervenciones eficaces y exitosas.

Los programas propuestos no han marchado por estar mal planteados: se carece de una definición de caso uniforme y clara, el enfoque prevaleciente es reduccionista al apegarse solo a la salud pública y no salir del esquema tradicional de prevención primaria, secundaria y terciaria ${ }^{5}$.

Esta revisión pretende ofrecer alternativas diferentes. En primer lugar, adoptar como definición de caso la del Centros para el Control y la Prevención de la Enfermedades (CCD) de Atlanta ${ }^{6}$; en segundo, ampliar la visión del asunto incluyendo aspectos filosóficos, psicosociales y psiquiátricos, y por último incluir como marco teórico el modelo psicológico «diátesis-estrés» ${ }^{7}$ proponiendo como estrategia preventiva «las intervenciones por poblaciones».

\section{La definición de caso de los CDC}

Esta concepción es la más aceptada y ecléctica. Describe con precisión los tres grados de la conducta suicida:

- Suicidio: muerte causada por conducta perniciosa autoinfligida, con la intención de quitarse la vida.

- Intento de suicidio: conducta autoinfligida, no mortal, potencialmente perniciosa, con la intención de quitarse la vida. Puede no producir daño.

- Ideación suicida: pensar, considerar o planear suicidarse.

\section{La visión sociocultural, filosófica y científica}

La visión del suicidio y la conducta suicida cambian según las diferentes culturas y épocas. Un ejemplo de ello sería Japón en contraste con los países de tradición judeocristiana. Para el primero, el suicidio es un acto de dignidad personal y orgullo nacional, como lo muestra el harakiri de los samuráis, vigente hasta nuestros días. Recordemos que el gran escritor Yukio Mishima ${ }^{8}$ lo llevó a cabo, en protesta por el sojuzgamiento de su nación a los EE.UU. Para la cultura judeocristiana, en cambio, se trata de un acto reprobable y condenable.
La percepción del suicidio cambia con las épocas. Durante el romanticismo estuvo bien visto cuando se llevaba a cabo por amor. Johann Wolfgang von Goethe, líder de la corriente romántica, publicó Las penas del joven Werther ${ }^{\ominus}$, cuyo personaje principal se quita la vida porque su amada no le corresponde. La novela fue un éxito mundial y tuvo tal repercusión que generó entre hombres y mujeres una ola de suicidios que llegó a alarmar a la sociedad ${ }^{10}$. Algo similar sucedió en nuestro medio en el caso de Manuel Acuña, poeta romántico y estudiante de medicina, que al quitarse la vida por el amor a Rosario ${ }^{11}$ desató una especie de moda de la muerte autoinfligida. $Y$ es que una de las consecuencias de la conducta suicida es la imitación; el sociólogo estadounidense David Phillips lo llamó en 1974 «el efecto Werther», en alusión a la célebre novela.

Un suicidio es un golpe para quienes rodean a la persona y para la sociedad en su conjunto, a la que llega a cimbrar. De ahí que se trate también de una poderosa herramienta política, como ilustra la emblemática autoinmolación del monje budista Thich Quang Duc ${ }^{12}$, quien se roció con gasolina y se prendió fuego en público para protestar por el trato que recibían los budistas del gobierno de Vietnam del Sur. La fotografía de su suicidio dio la vuelta al mundo y en cierto modo contribuyó al vuelco que daría la guerra de Vietnam.

La perspectiva filosófica de la conducta suicida muestra los distintos contrastes ante la existencia. Los cristianos la condenan, porque para ellos Dios es el dueño de nuestra vida y solo él puede disponer de ella. En cambio, hay quienes la consideran el máximo acto de libertad del hombre. El filósofo rumano Emil Cioran decía que «lo hermoso del suicidio es que es una decisión. Es muy halagador en el fondo poder suprimirse ${ }^{13}$.

La perspectiva científica de este tema se inicia en el en el siglo xix, con Émile Durkheim, sociólogo positivista y autor de El suicidio ${ }^{14}$, libro en el que sostiene que el acto suicida es un fenómeno social que rebasa al individuo. Él fue el primero en emplear las estadísticas para estudiarlo, pues no olvidemos que la epidemiología actual es heredera directa de Durkheim.

\section{Las visiones psicológica y psiquiátrica}

Abordar los múltiples ángulos que ofrecen la perspectivas psicológica y psiquiátrica ayuda a entender la conducta autodestructiva de las personas en tanto que individuos. 
Desde la perspectiva psicológica el acto de quitarse la vida puede leerse de diferentes maneras: una acción desesperada de quien no encuentra otra salida, un intento de comunicarse o de llamar la atención, una agresión con dedicatoria, una vía para descargar resentimientos y deseos hostiles. Para Freud, padre del psicoanálisis, todo suicidio es un homicidio frustrado ${ }^{15}$, es decir, lo realiza alguien que de manera inconsciente elige quitarse la vida, en lugar de quitársela a otro.

El inconsciente suicida también se manifiesta en las conductas de alto riesgo, sobre todo entre los adolescentes, en quienes no hay tal vez una intención autodestructiva, pero sí un reto o un desafío a la vida consistente en conducir autos a altas velocidades, practicar deportes de alta peligrosidad, etc. La búsqueda de emociones fuertes los acerca a la muerte y, entre más se acercan, más emocionante es la vida. Por eso no es de sorprender que el índice de mortalidad en este rango de edad sea más alto que en la población general, tal como lo demuestran también las primas de los seguros médicos y de vida ${ }^{16}$.

Una pregunta que suelen plantearse cuando una persona ha intentado suicidarse es qué tan serio fue el acto: ¿la persona realmente pretendió matarse 0 simplemente trató de llamar la atención? Pese a la complejidad de su respuesta, la pregunta parece necesaria para conocer mejor las motivaciones de la conducta suicida y para comprender a quienes la manifiestan. Para empezar, todo acto suicida es ambivalente ${ }^{17}$, ya que en el ser humano conviven fuerzas a favor de la vida y otras a favor de la muerte. Lejos del contraste blanco/negro, hay gradientes. Algunas personas están más resueltas que otras a quitarse la vida. El juego de la ruleta rusa es un buen ejemplo. En un revolver de seis balas los más decididos dejan solo un lugar vacío; los menos, dejan cinco o ninguno. No debe perderse de vista, por lo tanto, que las personas que deciden quitarse la vida, por más decididas que estén, siempre dejan abierto un resquicio que los pueda salvar.

Es por eso por lo que la pregunta sobre la seriedad de la intención de quitarse la vida debe de responderse con cuidado. Calificar el grado de «seriedad» es un asunto complejo y suele conllevar juicios de valor. Lo habitual es que la seriedad del acto se establezca mediante la letalidad del método elegido. Con esa lógica, por ende, los más serios son los que usan armas de fuego, se arrojan de lo alto de un edificio 0 se ahorcan. No obstante, la mayoría recurre a métodos en los que no es fácil calificar el grado de letalidad, tales como el monóxido de carbono del horno de la casa o del auto, cortarse las venas, ingerir sobredosis de medicamentos, etc. Para la correcta calificación del intento de suicidio es menester conocer en detalle los pormenores del acto y no limitarse simplemente a la letalidad del método. El juicio de valor que implica la pregunta a la que venimos refiriéndonos, por desgracia, divide a las personas en dos extremos: los intentos logrados llegan a ser vistos con respeto y aquellos que solo llaman la atención tienden a condenarse con severidad. De hecho, todos los suicidios, logrados o no, son como un grito de desesperación, una llamada de atención a los que rodean a quienes llegan a ese extremo. Por lo tanto, es recomendable no formular en primera instancia esa clase de pregunta y no perder de vista la angustia que está detrás de estas conductas.

Un aspecto psicológico también relevante para las personas que intentaron inútilmente quitarse la vida es el concepto de crisis $^{18}$. Esta se define como una desorganización mental debido a un problema que el individuo no es capaz de resolver mediante los métodos habituales. Se trata generalmente de una situación que lo sorprende o lo confunde profundamente y que puede convertirse en una encrucijada vital de dos caminos, hundirse o salir adelante. En otras palabras, un intento de suicidio bien sorteado y bien manejado por los profesionales y allegados del suicida puede convertirse en una oportunidad para enfrentar y resolver asuntos pendientes y conducir a un mayor crecimiento emocional. En cambio, si el intento se minimiza o no se trata adecuadamente, el resultado puede ser fatal.

Hay que tener presente que la mayoría de las personas que manifiestan conductas suicidas sufren 0 han sufrido previamente algún trastorno mental como depresión, bipolaridad, esquizofrenia, alcoholismo, consumo de drogas, etc. ${ }^{19}$. Así pues, el acto suicida es la exacerbación de una patología que viene de tiempo atrás. Con frecuencia hay intentos previos, y, en ciertos casos, antecedente de familiares que se quitaron la vida.

Un enfoque que ha ayudado a comprender a las personas que se han quitado la vida son las autopsias psicológicas, que consisten en reconstruir la conducta de estas personas los días previos a su muerte. Las autopsias psicológicas han mostrado que antes del evento hubo mensajes o avisos de alerta, que no fueron debidamente captados por las personas circundantes, incluidos los propios médicos y el personal de salud. Diferentes estudios demuestran que 
entre el 30 y el $50 \%$ de los suicidas había estado en contacto con algún médico o psiquiatra ${ }^{20}$. Este hallazgo ha estimulado la creación de instrumentos que intentan identificar el riesgo y prevenir las conductas suicidas. Sin embargo, ninguno ha resultado ser siempre del todo eficaz. Pese a la intervención preventiva, hay personas que terminan quitándose la ida. La experiencia demuestra, por lo tanto, que es más efectivo detectar la psicopatología que subyace en la intención y proceder a tratarla, advirtiendo siempre al interesado y a sus más allegados de la importancia de mantener una actitud vigilante.

\section{Un caso emblemático: Silvia Plath}

Uno de los casos más estudiados de suicidio es el de la poeta estadounidense Silvia Plath (1932-1963) ${ }^{21}$. Su caso ilustra con claridad lo aquí descrito. Su padre, con quien mantenía una estrecha relación, muere cuando ella tenía cinco años. A los 21 años (1953) ella presenta su primer intento suicida y es ingresada a un hospital psiquiátrico, donde recibe electrochoques y choques insulínicos, así como terapia psicoanalítica. En 1962 sufre un serio accidente automovilístico del que sale con vida, pero que ya puede catalogarse, yendo ella al volante, como conducta riesgosa. Ese mismo año se separa de su marido, el también poeta Ted Hughes (1930-1998). Paradójicamente, la separación coincide con un periodo de gran productividad literaria para ella, el cual, de acuerdo con su historial clínico, sugiere un episodio hipomaniaco, ya que a renglón seguido sufre el cuadro depresivo que desemboca en su suicidio el 11 de febrero 1963. No hay duda de que Silvia Plath sufría de un trastorno bipolar, que como se sabe tienen una fuerte carga genética; casi no es de extrañar que su hijo menor, nacido en 1962, se quitara la vida a los 47 años.

El suicidio de Silvia Plath parece no dejar ninguna duda de que ella estaba resuelta a morir. Metió la cabeza al horno de la estufa y lo prendió para inhalar el gas, un método bastante recurrente entre las suicidas en el Reino Unido. Sin embargo, analizándola con mayor detenimiento su decisión no resulta tan contundente. Sabía que en unos minutos llegaría, como todos los días, la puntual niñera de sus hijos, que ese día falló: una congestión del transporte público retardó su llegada. Cuando ingresó a la casa ella fue quien descubrió el cadáver.

El suicidio de Silvia tuvo una enorme repercusión. Su condición de mujer, su accidentada vida y su talento poético la convirtieron en un emblema para el movimiento feminista, que culpó de su muerte a Ted Hughes, por haberla abandonado por su mejor amiga. Condenado al ostracismo social, a Hughes lo rehabilitó la calidad de su propia obra poética.

\section{Un esquema preventivo diferente}

La referencia teórica más adecuada para guiar las intervenciones preventivas es el modelo psicológico "diátesis-estrés»" que se utiliza tanto en los trastornos mentales como en algunos fenómenos sociales ${ }^{22}$. La palabra diátesis se deriva del griego ( $\delta\llcorner\theta \varepsilon \varepsilon \sigma \iota)$, que significa predisposición, sensibilidad o debilidad. Este modelo es útil para explicar cómo interactúan los atributos biológicos o genéticos (diátesis) con las influencias ambientales (estresores) para producir un desorden como la conducta suicida ${ }^{22}$.

La estrategia preventiva de este enfoque en lugar de la tradicional primaria, secundaria y terciaria consiste en las llamadas intervenciones por población, que permiten diseñar acciones más precisas y efectivas. Dichas intervenciones se dirigen a tres tipos de poblaciones: a) la universal, que abarca a la población general; b) las selectivas, que se enfocan en grupos de alto riesgo, y c) las recomendadas, que no se dirigen a grupos, sino a individuos de muy alto riesgo.

\section{Intervenciones universales}

Este tipo de intervenciones están dirigidas a la población general en diferentes niveles (nacional, estatal o regional) y de grupos específicos (estudiantes, ancianos, enfermos, etc.). Las nacionales que han mostrado su eficacia aunque, cosa curiosa, no fueron diseñadas expresamente con el fin de prevenir el suicidio son: la restricción de compra y venta de armas de fuego, el uso de catalíticos automotrices para reducir la producción de monóxido de carbono, el cambio del gas doméstico convencional por gas natural. Otras medidas en este nivel y expresamente dirigidas al fin que nos ocupa son: la restricción del acceso a las azoteas de los edificios altos y la construcción de vallas protectoras en los puentes viales para evitar que las personas se arrojen desde allí. Con estas mismas intenciones se ha reducido el número tabletas en los envases de medicamentos controlados y su presentación ha cambiado a las burbujas de plástico. Si bien se trata de medidas 
inteligentes, no se ha establecido aún ninguna evidencia científica sobre su eficacia.

Otras estrategias preventivas nacionales consisten en dar acceso a la atención médica, mediante los servicios de salud pública, a las personas de alto riesgo, como son las que sufren trastornos mentales y/o consumen alcohol y drogas.

Las medidas enfocadas a sectores específicos de la población son las campañas educativas escolares para los alumnos y para que los maestros puedan identificar y ayudar a quienes se encuentran en crisis, así como las cruzadas para combatir el acoso escolar (bulling) que, aunque originalmente no se concibieron contra el suicidio, sí han ayudado a abatir las tasas de ese tipo de conducta en el estudiantado ${ }^{23}$.

\section{Las estrategias selectivas}

Este tipo de estrategias se dirigen a las poblaciones más vulnerables, entre las que se encuentran, además de los adictos, las personas recién diagnosticadas con una enfermedad grave, especialmente durante la tercera edad, los presos y muchos individuos en situación de calle.

El riesgo suicida de los enfermos mentales es diez veces mayor que en el resto de la población sea cual sea el tipo de padecimiento. Ya que muchos de estos trastornos no suelen identificarse $y$, por lo tanto, no se tratan en el primer nivel de atención, se han elaborado programas de capacitación para que los médicos generales reconozcan y traten a los enfermos con depresión. Se ha demostrado que con estos programas aumenta la prescripción de antidepresivos y bajan las tasas de conducta suicida.

El riesgo suicida aumenta entre quienes acaban de recibir el diagnóstico de alguna enfermedad grave, sobre todo neurológica o neoplásica. Por ello se aconseja evaluarlos con especial cuidado. Otras poblaciones de alto riesgo son las de las personas que viven en la calle, particularmente siendo jóvenes, así como los exprisioneros que acaban de ser puestos en libertad. Por desgracia, a estos grupos de riesgo no se les ha prestado la atención necesaria.

Otras intervenciones selectivas se recomiendan especialmente en el caso de las personas que han intentado suicidarse o han acudido a algún servicio médico por ideación suicida. En una revisión estadística se halló que el $16 \%$ de las personas que trataron de quitarse la vida lo volvieron a intentar en el curso de un año sin consecuencias fatales, pero que el $2 \%$ sí lo logró. Nueve años después, sin embargo, el 7\% lo logró ${ }^{24}$. Así pues, los intentos previos de suicidio, sobre todo cuando son repetitivos, constituyen el principal factor de riesgo y, por lo tanto, la señal de que se debe estar alerta.

En general, la mayor parte de las personas que intenta suicidarse suele ser llevada para su atención a los servicios de urgencias de los hospitales generales, donde lo ideal sería contar con un sistema adiestrado en el manejo de situaciones críticas. Pocas veces la intervención del personal médico en esa coyuntura tiene un resultado satisfactorio, por lo que es común que se dé de alta a la persona sin haber identificado el meollo de su problemática.

Por desgracia, hasta ahora no hay evidencia clara de cuál de estas intervenciones son útiles para la prevención. La que mejor parece funcionar es la terapia cognitiva conductual. Otras estrategias que han dado algunos resultados son dar seguimiento a los enfermos por medio de cartas periódicas o llamadas telefónicas.

\section{Conclusiones}

La prevención de conducta suicida puede ser efectiva si se adopta una definición de caso uniforme y ecléctica, se enfoca desde una perspectiva amplia que no se reduzca a la visión de la salud publica y se utilice un modelo intervencionista más operativo y adecuado a un fenómeno conductual tan complejo.

\section{Financiamiento}

El artículo no recibió ningún financiamiento.

\section{Conflicto de intereses}

Los autores declaran no tener ningún conflicto de intereses.

\section{Responsabilidades éticas}

Protección de personas y animales. Los autores declaran que para esta investigación no se han realizado experimentos en seres humanos ni en animales.

Confidencialidad de los datos. Los autores declaran que han seguido los protocolos de su centro de trabajo sobre la publicación de datos de pacientes.

Derecho a la privacidad y consentimiento informado. Los autores declaran que en este artículo no aparecen datos de pacientes. 


\section{Bibliografía}

1. Organización Panamericana de la Salud, Organización Mundial de la Salud. Prevención del suicidio: un imperativo global [Internet]. Organización Panamericana de la Salud, Organización Mundial de la Salud; 2014 [citado: 24 sep 2019]. Disponible en: https://www.paho.org/es/documentos/prevencion-suicidio-imperativo-global

2. Borges G, Nock MK, Medina-Mora ME, Benjet C, Lara C, Chiu WT, et al. The epidemiology of suicide-related outcomes in Mexico. Suicide Life Threat Behav. 2007;37(6):627-40.

3. Borges G, Orozco R, Villatoro J, Medina-Mora ME, Fleiz C, Díaz-Salazar J. Suicide ideation and behavior in Mexico: Encodat 2016. Salud Publica Mex. 2019;61(1):6-15.

4. Borges G, Benjet C, Orozco R, Medina-Mora ME. The growth of suicide ideation, plan and attempt among young adults in the Mexico City metropolitan area. Epidemiol Psychiatr Sci. 2017;26(6):635-43.

5. Wilcox HC, Wyman PA. Suicide prevention strategies for improving population health. Child Adolesc Psychiatr Clin N Am. 2016;25(2): 219-33.

6. Crosby AE, Ortega L, Melanson C. Self-directed violence surveillance. Uniform definitions and recommended data elements, Version 1.0 [Internet]. Atlanta, GA: Mational Center for Injury Prevention and Control, Division of Violence Prevention; 2011. Disponible en: http://www.cdc.gov/ violenceprevention/pdf/self-directed-violence-a.pdf

7. Hankin BL, Abela JRZ. Development of psychopathology: A vulnerability-stress perspective. Sage; 2005.

8. Mishima Yukio [Internet]. Encylopaedia Britannica; 2020. Disponible en: https://www.britannica.com/biography/Yukio-Mishima

9. Goethe J. Penas del joven Werther. Alianza Editorial; 2012.

10. Phillips D. Efecto Werther [Internet]. Wikipedia. Disponible en: https:// es.wikipedia.org/wiki/Efecto_Werther
11. Manuel Acuña, el último gran poeta del romanticismo en México: Marco Antonio Campos [Internet]. Gobierno de México, Secretaría de Cultura; 27 agosto 2013. Disponible en: https://www.gob.mx/cultura/prensa/manuel-acuna-el-ultimo-gran-poeta-del-romanticismo-en-mexico-marco-antonio-campos

12. Thich Quang Duc [Internet]. Wikipedia; 2020. Disponible en: https:// es.wikipedia.org/wiki/Thích_Quảng_Đức

13. Emil Cioran [Internet]. Wikiquote; 2020. Disponible en: https://es.wikiquote.org/wiki/Emil_Cioran

14. Durkheim E. El Suicidio (Spanish Edition). CreateSpace Independent Publishing Platform; 2017.

15. Ferreyra L. Suicidios y Freud: Conceptualizaciones sobre el suicidio en ensayos freudianos. Anu Investig. 2017;3(2):488-506.

16. Klonsky ED, Saffer BY, Bryan CJ. Ideation-to-action theories of suicide: a conceptual and empirical update. Curr Opin Psychol. 2018:22:38-43.

17. Kleiman EM, Nock MK. Real-time assessment of suicidal thoughts and behaviors. Curr Opin Psychol. 2018;22:33-7.

18. González de Rivera Revuelta JL. Psicoterapia de las crisis. Rev Asoc Española Neuropsiquiatría. 2001;79:37-51.

19. Robins E, Murphy GE, Wilkinson RH, Gassner S, Kayes J. Some clinical considerations in the prevention of suicide based on a study of 134 successful suicides. Am J Public Health Nations Health. 1959;49(7):888-99.

20. Turecki G, Brent DA. Suicide and suicidal behaviour. Lancet. 2016;387(10024):1227-39.

21. Alvarez A. The Savage God: A study of suicide. London: Pinguin; 1971.

22. Lazarus RS. From psychological stress to the emotions: A history of changing outlooks. Annu Rev Psychol. 1993;44:1-21.

23. Niederkrotenthaler T, Stack S, Till B, Sinyor M, Pirkis J, Garcia D, et al. Association of increased youth suicides in the United States with the release of 13 Reasons Why. JAMA Psychiatry. 2019;76(9):933-40.

24. Owens D, Horrocks J, House A. Fatal and non-fatal repetition of selfharm. Br J Psychiatry. 2002;181(3):193-9. 